N. ${ }^{\circ}$ 84, Julio - Diciembre, 2021 • pp. 132-159

ISSN: $1012-9790 \cdot$ e-ISSN: $2215-4744$

DOI: https://doi.org/10.15359/rh.84.7

http://www.revistas.una.ac.cr/historia

SECCIÓN COSTA RICA

\title{
Tendencias en la composición de la comunidad estudiantil de la Sede Regional Brunca de la Universidad Nacional (1973-2018)
}

Trends in the Composition of the Student Community of National University Brunca Branch (1973-2018) Tendências na composição da comunidade
estudantil da Sede Regional de Brunca da
Universidade Nacional (1973-2018) Edgardo Fonseca Zúñiga*

\begin{abstract}
Resumen: Este artículo presenta un análisis de la composición del estudiantado de la Sede Regional Brunca de la Universidad Nacional, con el fin de determinar la trayectoria histórica seguida por esta población beneficiada gracias al proceso de regionalización ejecutado por este centro de estudios. Dicho análisis procura vislumbrar, en alguna medida, la implementación de la óptica democratizante de esta universidad estatal. El argumento central de la investigación es el vínculo establecido entre el fortalecimiento institucional de la sede y la expansión de los servicios educativos que ofrece. Así, el crecimiento de la Sede Regional Brunca ha significado un mayor acceso a educación superior de grupos sociales vulnerables. Las fuentes utilizadas son las Estadísticas de Educación Superior del Consejo Nacional de Rectores y datos estadísticos de la Oficina de Registro de la Universidad Nacional.
\end{abstract}

Fecha de recepción: 17/03/2020 - Fecha de aceptación: 28/05/2020

* Costarricense. Estudiante del Posgrado de Historia en la Universidad de Costa Rica (UCR). Licenciado en Historia por la Universidad de Costa Rica (UCR), San José, Costa Rica. Docente e investigador en la Sede del Sur de la Universidad de Costa Rica (UCR), Golfito, Región Brunca, Costa Rica. Correo electrónico: edgardo.fonseca@ucr.ac.cr. 
Palabras clave: educación; enseñanza superior; universidades; democratización de la educación; historia de la educación.

\begin{abstract}
This article presents an analysis of the composition of the students of the National University Brunca Branch, in order to determine the historical trajectory followed by this population benefited by the regionalization process carried out by this study center. The intention of the analysis is to glimpse, to some extent, the implementation of the democratizing vision of this state university. The central argument of the research is the relationship established between the institutional strengthening of the Branch and the expansion of the educational services it offers. Thus, the growth of Brunca Branch has meant greater access to higher education for vulnerable social groups. The sources used are the Statistics of Higher Education of the National Council of Rectors and statistical data of the Registration Office of the National University.
\end{abstract}

Keywords: education; higher education; universities; democratization of education; educational history.

Resumo: Este artigo apresenta uma análise da composição do corpo discente da Sede Regional de Brunca da Universidade Nacional, com o objetivo de determinar a trajetória histórica percorrida por esta população beneficiada pelo processo de regionalização levado a cabo por este centro de estudos. A intenção desta análise é vislumbrar, em certa medida, a concretização da visão democratizante dessa universidade estadual. $\mathrm{O}$ argumento central da pesquisa é a relação que se estabelece entre o fortalecimento institucional da sede e a ampliação dos serviços educacionais por ela oferecidos. Assim, o crescimento da Sede Regional de Brunca significou um maior acesso ao ensino superior para grupos sociais vulneráveis. As fontes utilizadas são Estatísticas do Ensino Superior do Conselho Nacional de Reitores e dados estatísticos do Cartório de Registro da Universidade Nacional.

Palavras chaves: educação; ensino superior; universidades; democratização da educação; história da educação.

\title{
Introducción ${ }^{1}$
}

La regionalización del sector educativo superior en Costa Rica inicia en el contexto de expansión de tal área formativa, registrado en la década de 1970, el cual impulsó el modelo desarrollista promovido por los gobiernos de la época. ${ }^{2}$ Tuvo sus comienzos en 1968 en la Universidad de Costa Rica (UCR), con la

1 Este artículo presenta los principales resultados del proyecto de investigación B9138 Regionalización de la educación superior en Costa Rica, el caso de la Sede Regional Brunca de la Universidad de la Universidad Nacional, adscrito a la Vicerrectoría de Investigación de la Universidad de Costa Rica y, que tiene como unidad ejecutora la Sede del Sur de la Universidad de Costa Rica.

2 Iván Molina Jiménez, La educación en Costa Rica. Desde la época colonial hasta el presente (San José, Costa Rica: EDUPUC, 2016), 394 y ss. 
creación del Centro Regional de San Ramón. Posteriormente, fueron establecidas otras sedes en Liberia, Turrialba, Limón y Puntarenas. ${ }^{3}$ En el caso de las otras universidades estatales, el Instituto Tecnológico (TEC) asumió la administración, como sedes, de la Escuela Técnica Agrícola de Santa Clara en San Carlos y la Escuela Técnica Nacional en San José desde su fundación en $1973 .{ }^{4}$ La Universidad Nacional (UNA) fue constituida en 1973, tomando como base la Escuela Normal de Heredia y sus sedes en Liberia, San Ramón y Pérez Zeledón. ${ }^{5}$

Este fenómeno de regionalización universitaria también alcanzó a la Región Brunca. Localizada en el sur de Costa Rica, esta se extiende desde el Valle de El General hasta la frontera con Panamá. Está conformada por 41 distritos distribuidos en 6 cantones. Uno de estos, Pérez Zeledón, se encuentra en la provincia de San José. Los restantes 5, Buenos Aires, Osa, Golfito, Coto Brus y Corredores, se ubican en la provincia de Puntarenas.

En la región, existe la oferta de 3 universidades públicas: la UNA, en Pérez Zeledón y Corredores; la UCR, en Golfito, y la Universidad Estatal a Distancia (UNED), distribuida en todos los cantones. Además, se hallan 6 universidades privadas concentradas, principalmente, en Pérez Zeledón. ${ }^{6}$ En total, 10 instituciones brindan sus servicios en 20 campus, con una oferta académica de 8 diplomados, 32 bachilleratos, 31 licenciaturas y 8 posgrados para el $2015^{7}$ Estas casas de estudio ofrecen carreras relacionadas con educación, inglés, turismo y negocios.

Específicamente, la UNA inició operaciones en Pérez Zeledón con un centro regional, desde su creación, en 1973, hasta 1991, cuando en el Acta 1387 del 21 de marzo el Consejo Universitario acuerda su reorganización como una sede universitaria con el nombre de Sede Regional Brunca. ${ }^{8}$ Los argumentos dados por las autoridades resaltan la evolución del centro como polo de desarrollo y el éxito alcanzado en la ejecución de programas docentes, extensión universitaria e investigación. Además, se consideró la desconcentración administrativa ejecutada por la UNA desde 1988, así como la recomendación del Congreso Universitario de transformar el centro en sede y brindarle mayor autonomía. Actualmente, la Sede Regional Brunca opera con 2 campus, uno en Pérez Zeledón y otro en Corredores, el cual cuenta con instalaciones propias desde el 2004.

El Estatuto Orgánico de la UNA, en su artículo 2, define los valores que rigen a esta institución de estudios superiores. Se destacan el compromiso social,

3 Edgardo Fonseca Zúñiga, «La regionalización de la educación superior en Costa Rica, el caso de la Universidad de Costa Rica en Golfito, 1984-2017», Revista Estudios, n³8 (junio-noviembre 2019): 5, https:// revistas.ucr.ac.cr/index.php/estudios/article/view/37463/38268.

4 Molina Jiménez, La educación..., 395.

5 Ibíd.

6 Programa Estado de la Nación, V Estado de la Educación (San José, PEN, 2015), 241-242.

7 Ibíd.

8 Marvin Cheng Ta, et al., «La política de regionalización en la educación superior estatal» (Tesis de Licenciatura en Administración Pública, Universidad de Costa Rica, 1994), anexo 18. 
entendido como la «consecución de una mejor calidad de vida para los sectores sociales menos favorecidos», y la equidad, procuradora de que todos los integrantes de la comunidad universitaria tengan «los mismos derechos y oportunidades». Igualmente, el artículo 3, que contiene los fines institucionales, refiere a la regionalización como un instrumento con el que la universidad «aporta al desarrollo del país». ${ }^{9}$ De esta manera, la UNA se configura como un sitio formador, que busca democratizar el acceso a educación superior en el país y su programa de regionalización, como un espacio para cumplir con este propósito.

La Sede Regional Brunca, parte del programa de regionalización, opera en una de las zonas con menor desarrollo social del país. Esta es la región socioeconómica y de planificación que presenta mayores índices de pobreza y desigualdad. El 88 \% de sus distritos se encuentra en los quintiles I y II del Î́ndice de Desarrollo Social (IDS), los más bajos. ${ }^{10}$ Esto se traduce en escolaridad baja, problemas de seguridad ciudadana, escasos ingresos en el hogar y pobreza.

Ante este panorama, la importancia de esta investigación radica en observar el papel democratizador realizado por la UNA, a través de la operación de una de sus sedes regionales en una de las zonas menos desarrolladas del país. Así se puede medir, en algún grado, la tarea llevada a cabo por esta universidad estatal, en la expansión del acceso a educación superior. Esto se logra al determinar la trayectoria histórica seguida por la población que ha disfrutado de los servicios educativos ejecutados por dicha institución.

De esta manera, el objetivo de la investigación es analizar la forma en la que se compone la comunidad estudiantil de la Sede Regional Brunca de la UNA, para establecer la trayectoria histórica de aquella que ha sido beneficiada con el proceso de regionalización llevado a cabo por tal universidad en esta zona. Lo anterior, para vislumbrar, en alguna medida, cómo ha sido implementada esta visión democratizante configurada en el estatuto de la UNA. Para lograrlo, primero se describe la matrícula, con el propósito de medir el peso de la regionalización en el proceso evolutivo de la UNA y las particularidades manifestadas en la Sede Regional Brunca. Seguidamente, se caracteriza a los estudiantes, según indicadores sociales y económicos, con el fin de perfilar quiénes han sido favorecidos con este procedimiento regionalizador. Finalmente, se particulariza a la población de graduados, con el objetivo de observar las oportunidades académicas ofrecidas en esta sede y perfilar a las personas que las han disfrutado.

\section{Metodología}

Los datos fueron extraídos y tratados para construir series estadísticas, las cuales son presentadas en cuadros y gráficos. Se intenta reconstruir la evolución de los indicadores representados e identificar las continuidades, rupturas y tendencias que esos datos muestran sobre la composición de las poblaciones estudiadas. Debe advertirse que la información no se halló uniformemente para 
todo el lapso propuesto. Por ello, en la reconstrucción del trayecto seguido por estudiantes y graduados existen periodos con más detalle que otros. Particularmente, el intervalo 2007-2018 es el que cuenta con datos más precisos.

La matrícula es estudiada según cantidad, distribución por sedes, admisión y distribución por área temática. Los estudiantes son analizados de acuerdo con sexo, edad, cantón, distrito y zona de procedencia, IDS del distrito de origen y condición de becado. Finalmente, la población de graduados se observa conforme títulos otorgados, distribución por grado, sexo y área temática del certificado.

Las fuentes utilizadas en esta indagación son principalmente dos de naturaleza estadística. La primera es la colección de Estadísticas de la Educación Superior, publicadas por el Consejo Nacional de Rectores (CONARE). Estas se editan desde 1978 hasta su última aparición publicada en 1999. De aquí se extrajeron datos referidos a matrícula, admisión y graduaciones.

La otra fuente empleada corresponde a tres planillas en formato Excel, proporcionadas por la Oficina de Registro de la UNA. Estas se llaman Admisión, Graduación y Matrícula de la Sede Brunca 2007-2018. Cabe destacar que en dichas planillas es posible distinguir la información de los dos campus que conforman la sede. Asimismo, se usaron otras fuentes de la Oficina de Registro de la UNA, disponibles en la página web Estadísticas Estudiantiles de la Universidad Nacional, para complementar la información de estudiantes y graduados. ${ }^{11}$

Por último, este centro de estudios ha tenido distintos nombres durante su funcionamiento; inició como la Escuela Normal de Pérez Zeledón, operó luego como el Centro Regional de Pérez Zeledón y se reorganizó como Sede Regional Brunca a partir de 1991. Con el fin de facilitar la rotulación de los datos, se optó por llamarlo Sede Regional Brunca, durante todo el periodo estudiado.

\section{Descripción de la matrícula}

En esta primera sección, se describe la matrícula reportada en la UNA y en la Sede Regional Brunca desde 1973 hasta el 2019. Esto con el propósito de medir el peso de la regionalización en el proceso evolutivo estudiantil de esta universidad. Además, se observa la cantidad y distribución, según la oferta académica ofrecida, de la matrícula de la Sede Regional Brunca. La idea fue identificar las particularidades en el recorrido de esta sede.

Desde su fundación en 1973, la UNA distribuyó sus servicios en un campus central, Omar Dengo, en Heredia, y tres sedes regionales en San Ramón, Pérez Zeledón y Liberia. En 1975, en un proceso no exento de polémica, la sede ramonense fue absorbida por el Centro Universitario de la UCR. ${ }^{12}$

La distribución de la matrícula por sede puede brindar datos sobre la fase evolutiva del proyecto de regionalización de esta universidad, al observar el peso

11 Esta página puede ser accedida a través de la dirección https://www.registro.una.ac.cr/see/

12 Centro Universitario de San Ramón, Informe de Labores 1975-1976 (Manuscrito inédito, s. f.), 3. 
que este ha tenido en la población estudiantil a lo largo de los años. Las pesquisas para el periodo de 1974 a 2017 se muestran en el siguiente gráfico:

Gráfico 1. Universidad Nacional. Distribución porcentual de la matrícula por sede (1974-2017)

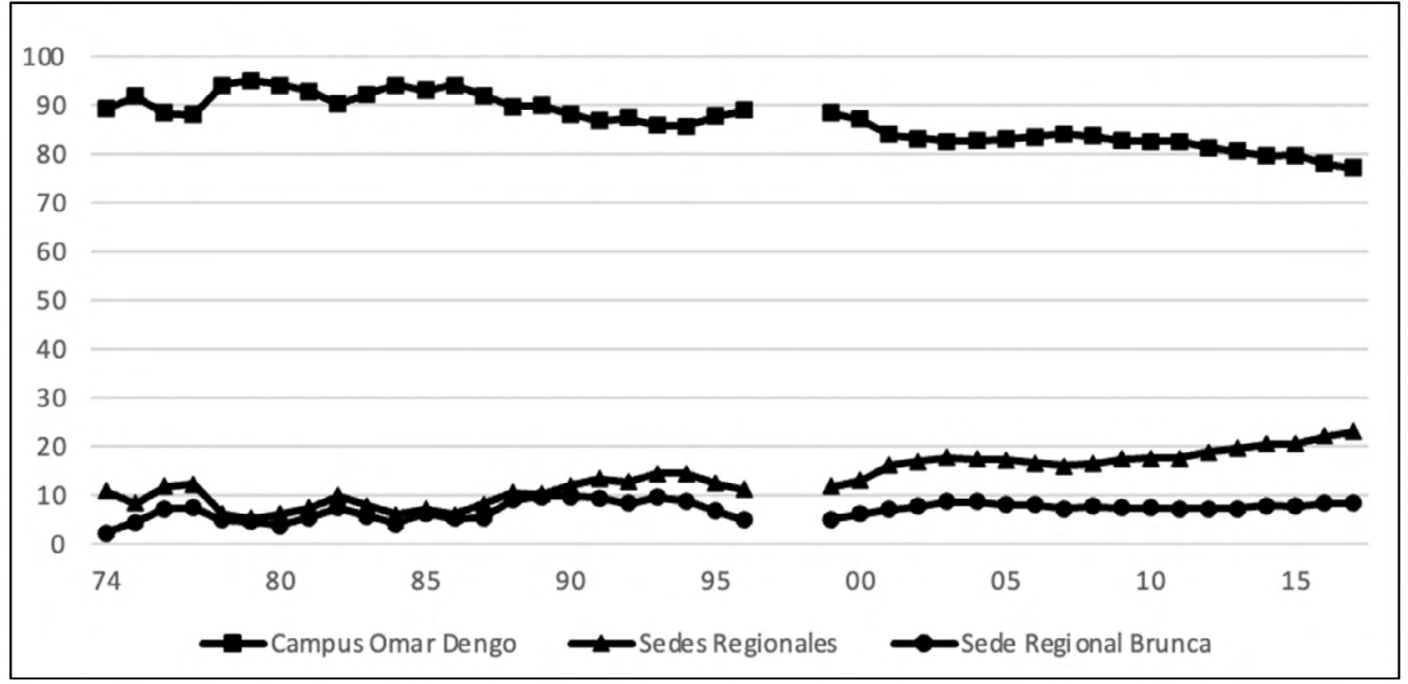

Fuente: Elaborado a partir de datos de CONARE, Estadística de la Educación Superior, 1978, cuadro 1.1 .1 , pág. 20; 1980 a, cuadro 1.1 .1 , pág. $16 ; 1980$ b, cuadro 1.1 .1 , pág. $24 ; 1981$, cuadro 1.1.1, pág. 26; 1982, cuadro 1.1.1, pág. 24; 1983, cuadro 1.1.1, pág. 25; 1984, cuadro 1.1.2, pág. 18 ; 1985, cuadro 1.1.1, pág. $11 ; 1986$, cuadro 1.1.2, pág. 14 ; 1987, cuadro 1.1 .2 , pág. 10; 1988, cuadro 1.1.2, pág. 10;1989, cuadro 1.1.2, pág. 9; 1991b, cuadro 1.1.2, pág. 11; 1992, cuadro 1.1.2, pág. 6; 1994, cuadro 1.1.2, pág. 6; 1996, cuadro 1.1.2, pág. 9; 1999, cuadro 1.1.2, pág. 7. Matrícula del primer periodo lectivo de las instituciones de educación superior universitaria estatal, por institución y sede. 2000-2017.

El porcentaje de participación de las sedes regionales en el total de la matrícula fue creciente en la primera década de existencia de la UNA, al menos hasta 1977. Posiblemente se trate de alumnos matriculados en los centros regionales de la Escuela Normal, quienes se mantuvieron como estudiantes de la nueva universidad. Este comportamiento está en sintonía con la expansión de la matrícula en este nivel educativo, experimentada en el país durante la década aludida.

A partir de 1978, el porcentaje de alumnos regionalizados decrece, con los puntos más bajos en la década de 1980. Igualmente, la segunda mitad de la década de 1990 también reporta una disminución del porcentaje de estudiantes regionalizados. Ambos fenómenos deben contextualizarse en la situación global educativa del país para esos años. La contracción de matrícula de la década de 1980 coincide con la crisis económica acontecida en ese momento. Esta significó 
una mengua en la inversión educativa y un estancamiento en el presupuesto asignado a la formación superior pública. ${ }^{13}$ Sumado a lo anterior, tanto la matrícula como la cobertura en este nivel educativo disminuyeron considerablemente durante la mencionada crisis. ${ }^{14}$ El desfinanciamiento de la educación superior estatal continuó para la década de 1990. Esto implicó que las universidades aplicaran la estrategia de congelar o disminuir la matrícula. ${ }^{15}$

Un fenómeno similar al observado en la UNA ocurrió en las sedes regionales de la UCR, las cuales también disminuyeron su matrícula durante la crisis de 1980. ${ }^{16}$ Entonces, ¿el contexto antes descrito puede explicar la concentración de alumnos en los campus centrales y su disminución en las sedes regionales, como una política universitaria para optimizar recursos y enfrentar dicha situación? Aunque se debe realizar una investigación más profunda para responder esta interrogante, es preciso advertir que en la UCR algunos docentes denunciaban esta aminoración de recursos, que se refleja en una oferta académica limitada y la restricción de presupuesto para la regionalización. ${ }^{17}$ Los datos de la UNA sugieren que pudo ocurrir algo parecido en esta universidad.

Hacia las dos primeras décadas del siglo XXI, la participación de las sedes regionales aumenta significativamente y a partir de 2007 entra en un periodo de crecimiento. Este comportamiento se vio influenciado por la apertura de una nueva sede de la UNA en Limón y su incursión en el proyecto Intersedes en Alajuela. Lo anterior se dio en un contexto de expansión vivido por las universidades públicas, beneficiado en gran medida por un aumento presupuestario que se concedió a través del Fondo Especial para la Educación Superior (FEES). ${ }^{18}$ Este fenómeno de expansión regional es similar para la UCR: para el 2017, una cuarta parte de sus estudiantes pertenecía a una sede regional. ${ }^{19}$ Con los datos anteriores, se puede afirmar que la segunda década del siglo XXI inaugura el periodo más favorable para la regionalización universitaria estatal en la historia costarricense.

Para el caso específico de la Sede Regional Brunca, su porcentaje en el total de la matrícula varía sustancialmente durante todo el periodo en estudio. La mayor participación se da entre 1989 y 1993, lo que parece justificar la decisión de transformarla en sede regional, justamente en 1991. Conforme se dio la apertura de otras sedes regionales, la incursión de la Sede Regional Brunca fue disminuyendo. Ya para las dos primeras décadas del siglo XXI estabiliza su participación de entre 7 y $8 \%$ del total de estudiantes matriculados en la UNA.

13 Molina Jiménez, La educación..., 442.

14 Ibíd., 465.

15 Molina Jiménez, La educación..., 537-538.

16 Ibíd., 471.

17 Yolanda Dachner Trujillo, «La Regionalización de la Universidad de Costa Rica, una propuesta para avanzar» (Ponencia presentada en el IV Seminario Carlos Monge Alfaro-La universidad pública en el mundo contemporáneo, Costa Rica, 4, 5 y 6 de noviembre de 1998), 70.

18 PEN, 2013, 226.

19 Fonseca Zúñiga, «La regionalización...», 6. 
La distribución de los alumnos admitidos en el programa de regionalización de la UNA se observa en el gráfico que se presenta a continuación:

Gráfico 2. Universidad Nacional. Distribución porcentual de admisión por Sedes Regionales y Sede Brunca (1977-2019)

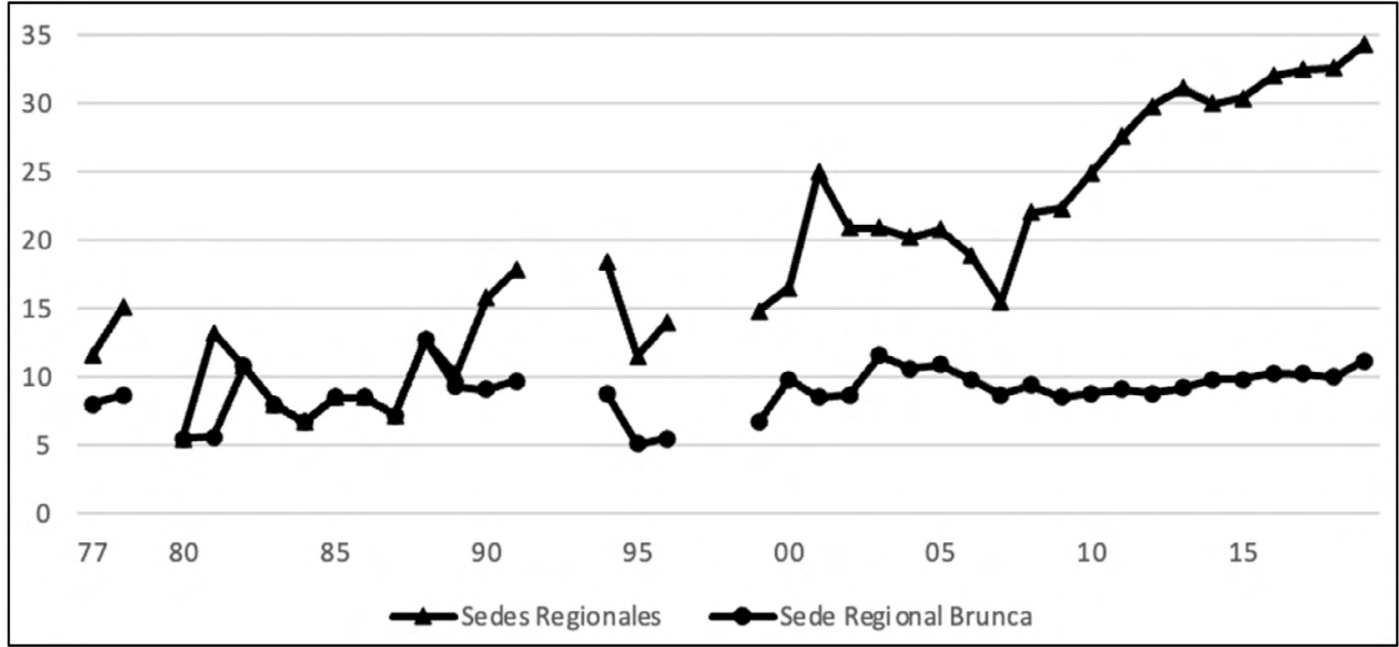

Fuente: Elaborado a partir de datos de CONARE, Estadística de la Educación Superior, 1978, cuadro 1.2 .6 , pág. $35 ; 1981$, cuadro 1.2 .5 , pág. 52 ; 1982, cuadro 1.2 .4 , pág. 42 ; 1984, cuadro 1.2.2, pág. $52 ; 1985$, cuadro 1.2 .1 , pág. $36 ; 1986$, cuadro 1.2 .3 , pág. $49 ; 1987$, cuadro 1.2 .2 , pág. 38; 1988, cuadro 1.2.2, pág. 43; 1989, cuadro 1.2.2, pág. 43; 1991b, cuadro 1.2.1, pág. 48; 1992, cuadro 1.2.2, pág. $38 ; 1994$, cuadro 1.2.2, pág. $38 ; 1996$, cuadro 1.1.2, pág. 9; 1999, cuadro 1.2.2, pág. 31. Oficina de Registro UNA. Cuadro 2.11. Cantidad de estudiantes de primer ingreso matriculados por facultad, centro y sede. Periodo 2000-2015. Oficina de Registro UNA, Admisión Sede Brunca 2007-2018.

Los dos últimos años de la década de 1970 muestran una participación considerable de admitidos en sedes regionales, con respecto al total de la UNA. Esta incursión bajó en la década siguiente, años en los cuales las fuentes consultadas solamente reportan admisiones en Pérez Zeledón, y vuelve a crecer hacia inicios de la década de 1990; aun así, cae una vez más a la mitad de esta última. Finalmente, a partir del 2008, se muestra un aumento importante de la participación de admitidos por el sistema de regionalización; en el 2019, estos representan el 34,3\% de todos los estudiantes nuevos de la UNA.

En lo que respecta específicamente a la Sede Regional Brunca, esta fue prácticamente la única opción regional en gran parte de la década de 1980. Partiendo del 2008, esta sede consolida su porcentaje de participación en el total de alumnos nuevos de la UNA; se ubica entre 9 y $10 \%$.

La distribución específica de la matrícula en la Sede Regional Brunca se muestra en el siguiente gráfico: 
Gráfico 3. Sede Regional Brunca. Distribución total de matrícula por campus (1973-2018)

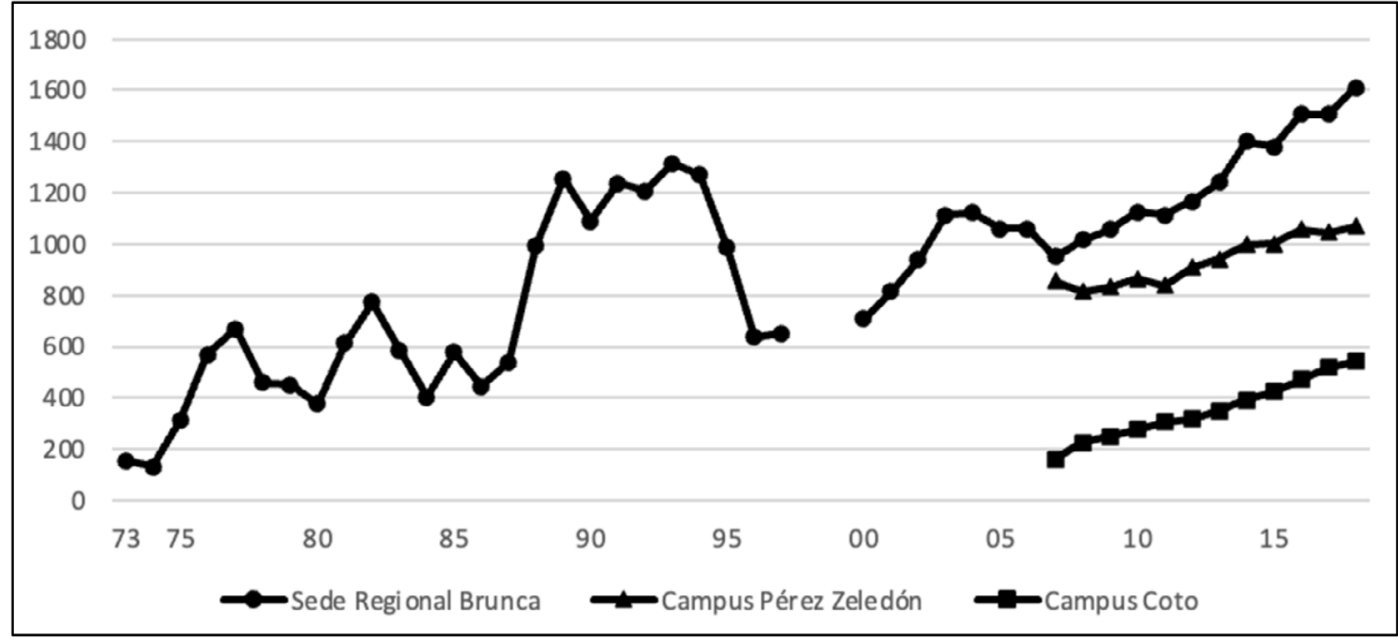

Nota: Las fuentes consultadas permiten realizar una distinción entre los matriculados en el Campus Pérez Zeledón y el Campus Coto, a partir del 2007.

Fuente: Elaborado a partir de datos de CONARE, Estadística de la Educación Superior, 1978, cuadro 1.1.1, pág. 20; 1980a, cuadro 1.1.1, pág. 16; 1980b, cuadro 1.1.1, pág. 24; 1981, cuadro 1.1.1, pág. 26; 1982, cuadro 1.1.1, pág. 24; 1983, cuadro 1.1.1, pág. 25; 1984, cuadro 1.1.2, pág. 18; 1985, cuadro 1.1 .1 , pág. $11 ; 1986$, cuadro 1.1 .2 , pág. 14 ; 1987, cuadro 1.1 .2 , pág. 10; 1988, cuadro 1.1 .2 , pág. $10 ; 1989$, cuadro 1.1 .2 , pág. $9 ; 1991$ b, cuadro 1.1 .2 , pág. $11 ; 1992$, cuadro 1.1.2, pág. 6 ; 1994, cuadro 1.1.2, pág. 6 ; 1996, cuadro 1.1.2, pág. $9 ; 1999$, cuadro 1.1.2, pág. 7. CONARE. Matrícula del primer periodo lectivo de las instituciones de educación superior universitaria estatal, por institución y sede. 2000-2017. Oficina de Registro UNA, Matrícula Sede Brunca 2007-2018.

Lo primero por destacar es la trayectoria heterogénea presentada por la matrícula de este centro regional durante todo el periodo de estudio. Etapas de importante crecimiento conviven junto a disminuciones considerables, con lo cual la matrícula muestra un comportamiento bastante irregular, lo que puede sugerir un complejo proceso de consolidación de este centro de estudios. Así, la década de 1970 presenta un crecimiento hasta 1977, bajando a partir de ese año. La década de 1980 deja ver un aumento importante hacia 1982, pero nuevamente cae. Desde 1988 y hasta 1994, se reporta otro importante incremento, pero la matrícula se desploma desde la segunda mitad de esta década y hasta el inicio del siglo XXI. A partir de 2008, inicia un acrecentamiento que se ha mantenido; en el 2014, llega a superar la cifra máxima histórica que se había registrado 21 años antes.

La otra característica que muestra este gráfico es el papel jugado por el Campus Coto. Desde el 2007, el primer año que nos brinda datos, se ha registrado 
un robustecimiento notable. Así, dicho año representaba el 15,9 \% del total de alumnos de la sede; una década después, el 33,7\%, un agrandamiento de más del doble. De esta forma, el Campus Coto tiene una participación considerable en las cifras de matrícula registradas en la segunda década del siglo XXI.

El análisis conjunto de los tres gráficos anteriores permite concluir al menos dos características del sistema de regionalización de la UNA y su reflejo en la Sede Regional Brunca. Primero, las irregularidades presentadas en la matrícula sugieren que el proceso de consolidación del modelo de regionalización se ajustó por completo al contexto educativo y económico del país. Periodos de desfinanciamiento y estrechez presupuestaria coinciden con una disminución del peso de las sedes regionales en el total de la matrícula, lo que supone que, ante presupuestos limitados, la prioridad se colocó en la sede central. Una expansión definitiva de la regionalización de esta universidad se logra a partir de la segunda década del siglo XXI, con un ambiente político y financiero favorable.

En segundo lugar, la reorganización del Centro Regional de Pérez Zeledón como Sede Regional Brunca no se tradujo inmediatamente en un fortalecimiento, al menos en matrícula, de esta institución. Al contrario, después de 1991, la recién bautizada sede enfrentó un proceso de caída en la cantidad de estudiantes que atendía y su recuperación se logró hasta ya entrado el siglo XXI. Así, en el 2008, 17 años después de la reestructuración de la Sede, es que logra un crecimiento sostenido del estudiantado. Otro aspecto importante para el estudio de la matrícula es la distribución por áreas temáticas. Su análisis puede dar pistas acerca de la oferta académica del centro y las carreras a las cuales pueden acceder los estudiantes de esta región. Los resultados obtenidos se presentan en el siguiente cuadro:

Cuadro 1. Sede Regional Brunca. Distribución porcentual de la matrícula por área temática (1982-2018)

\begin{tabular}{|l|l|c|c|c|c|}
\hline & Letras & Turismo & $\begin{array}{l}\text { Ciencias e } \\
\text { Ingeniería }\end{array}$ & Educación & $\begin{array}{l}\text { Sociales y } \\
\text { Economía }\end{array}$ \\
\hline $\mathbf{1 9 8 2}$ & & & & 21,6 & \\
\hline $\mathbf{1 9 8 3}$ & & & & 44,4 & \\
\hline $\mathbf{1 9 8 4}$ & & & & 49,9 & \\
\hline $\mathbf{2 0 0 7}$ & & 11,4 & 13,3 & 51,8 & 23,5 \\
\hline
\end{tabular}




\begin{tabular}{|l|l|l|l|l|l|}
\hline $\mathbf{2 0 0 8}$ & 6,3 & 16,2 & 18,1 & 38,0 & 21,4 \\
\hline $\mathbf{2 0 0 9}$ & 6,4 & 20,1 & 18,7 & 32,0 & 22,8 \\
\hline $\mathbf{2 0 1 0}$ & 7,2 & 19,3 & 22,2 & 23,9 & 27,4 \\
\hline $\mathbf{2 0 1 1}$ & 7,0 & 17,7 & 23,0 & 22,5 & 29,8 \\
\hline $\mathbf{2 0 1 2}$ & 7,3 & 14,7 & 23,7 & 23,8 & 30,5 \\
\hline $\mathbf{2 0 1 3}$ & 9,1 & 12,6 & 27,8 & 20,0 & 30,5 \\
\hline $\mathbf{2 0 1 4}$ & 7,8 & 12,0 & 27,3 & 19,4 & 33,5 \\
\hline $\mathbf{2 0 1 5}$ & 6,4 & 11,6 & 27,0 & 17,7 & 37,3 \\
\hline $\mathbf{2 0 1 6}$ & 5,8 & 10,4 & 26,1 & 17,7 & 40,0 \\
\hline $\mathbf{2 0 1 7}$ & 4,2 & 9,8 & 25,5 & 18,3 & 42,2 \\
\hline $\mathbf{2 0 1 8}$ & 4,5 & 8,6 & 25,0 & 21,3 & 38,4 \\
\hline
\end{tabular}

Fuente: Elaborado a partir de datos de CONARE, Estadística de la Educación Superior, 1983, cuadro 1.1.4, pág. 31; 1984, cuadro 1.1.6, pág. 32; 1985, cuadro 1.1.5, pág. 23. Oficina de Registro UNA, Matrícula Sede Brunca 2007-2018.

Lo primero que debe mencionarse es el considerable número de estudiantes que matriculaban lo conocido como Propedéuticos, para obtener un Certificado de Humanidades, durante la década de 1980. Las fuentes consultadas no los ubican en ninguna carrera, por lo cual se presume que eran alumnos quienes tomaban estos cursos humanísticos como requisito previo para, posteriormente, empadronarse en cualquier otra carrera ofrecida por la UNA. Los educandos en esa condición representaron el 63,5\% de la matrícula en 1982, el 45,1\% en 1983 y el $41,9 \%$ en $1984 .{ }^{20}$ Esto parece indicar que un porcentaje importante de estudiantes tomaban la sede como un espacio donde podían completar un requisito, como dichos cursos propedéuticos, sin matricular ninguna las carreras ofrecidas en el centro. Probablemente, de ahí se trasladaban a otra sede universitaria con una oferta académica más atractiva para sus intereses.

Esta estrategia parece que también fue seguida por estudiantes de las sedes regionales de la UCR en la década de 1970, quienes tomaban los cursos de educación básica sin estar necesariamente matriculados en alguna carrera. ${ }^{21}$ ¿Existía alguna relación entre los Propedéuticos de la UNA y los Estudios Generales de la UCR? ¿Eran cursos que podían convalidarse entre ambas universidades? No poseemos información que permita responder contundentemente a estas preguntas, pero la disponible permite al menos considerar la posibilidad de la utilización de este centro regional como un espacio donde aplicar tal estrategia. Con los

20 Consejo Nacional de Rectores (CONARE), Estadística de la Educación Superior (San José, Costa Rica: CONARE, 1983), 25; 1984, 18; 1985, 11.

21 Edgardo Fonseca Zúñiga, «Las Humanidades en la génesis de la regionalización universitaria: los Estudios Generales y el origen del proceso de regionalización de la Universidad de Costa Rica», Revista Estudios, número especial (2020): 27, https://revistas.ucr.ac.cr/index.php/estudios/article/view/40925/41537. 
datos analizados, se observa que la tendencia decreció al avanzar los años, aun así, es imposible determinar cuándo menguó por completo.

La otra característica relevante para la década de 1980 es la progresiva importancia tomada por las carreras en educación. La información al alcance indica que este tipo de carreras fue prioritario, pues concentra la mayor cantidad de la oferta académica. En la misma época, ocurría un fenómeno similar en las sedes regionales de la UCR, las cuales también presentaban una predilección por carreras educativas. De este modo, el comportamiento permite insinuar que la regionalización universitaria se orientaba a especializarse en educación, siendo parte de un plan que intentaba paliar, en las distintas regiones del país, la creciente demanda de educadores. $^{22}$

En el 2008 inicia una diversificación temática más pronunciada en la oferta académica de la Sede Brunca. Aun así, esta diversidad temática se apoyó en la oferta de una o dos carreras por área. El mejor ejemplo se encuentra en Ciencias e Ingeniería, que aumentan, considerablemente, su participación en la matrícula basada en una sola carrera: Informática. El área de Sociales y Economía sufre un comportamiento similar, al alimentar su progresiva importancia en una carrera de Administración y en otra de Comercio y Negocios Internacionales.

Comparada con los datos del gráfico 2 , se observa una mayor diversificación temática de la oferta académica que sintoniza con un aumento de matrícula. Así, a una mayor cantidad de estudiantes corresponde una oferta más variada de opciones de carrera.

\section{Distribución socioeconómica de la población estudiantil}

En esta segunda parte de la investigación, se expone la distribución de la comunidad estudiantil de la sede, según criterios socioeconómicos relacionados con sexo, edad, procedencia geográfica y condición de beca. Esto se hace con el propósito de perfilar quiénes han sido beneficiados con el proceso de regionalización. Igualmente, puede medirse, en algún grado, el alcance de esta identificando a las personas que impacta.

Lo primero que se presenta es la distribución del estudiantado por sexos. Poseemos información desde el 2007, mostrada en el siguiente gráfico:

Gráfico 4. Sede Regional Brunca. Distribución porcentual de la matrícula por sexo (2007-2018) 


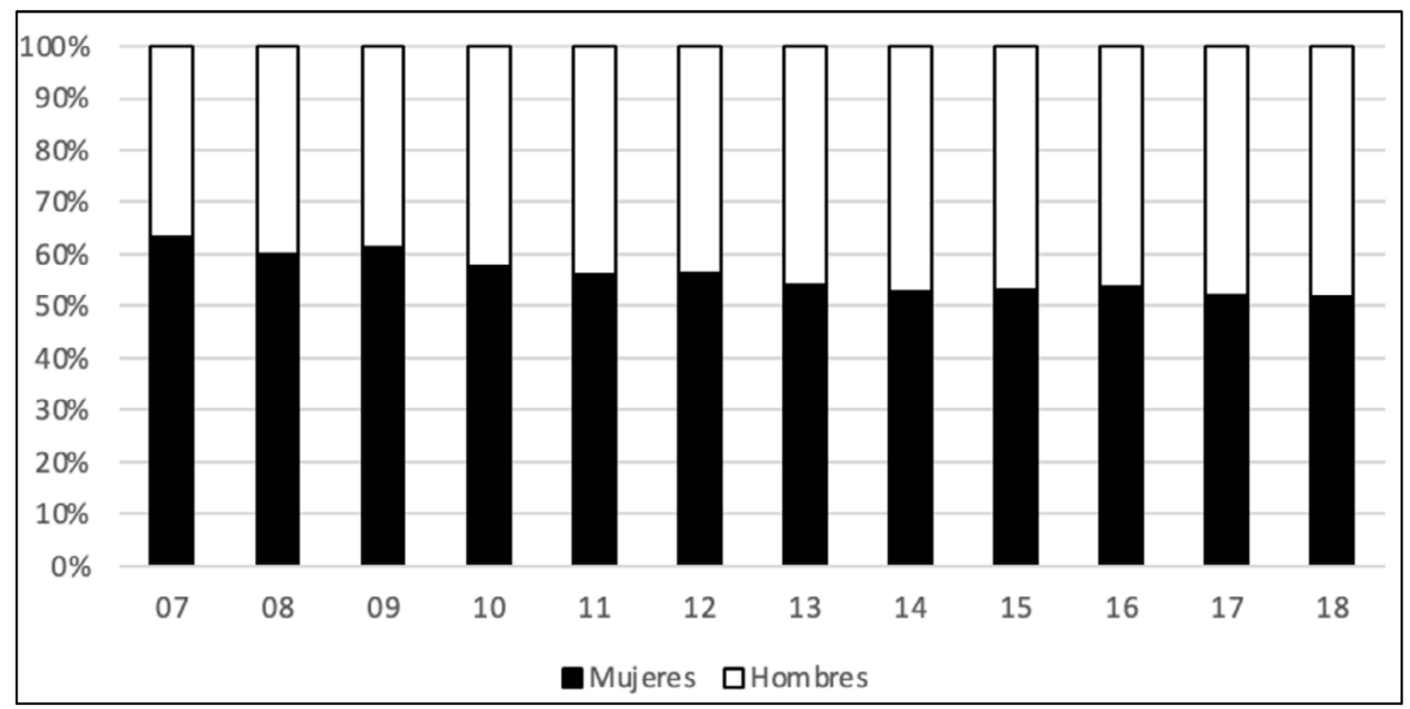

Fuente: Elaborado a partir de datos de la Oficina de Registro UNA, Matrícula Sede Brunca 2007-2018.

Durante todo el periodo analizado, el gremio estudiantil está compuesto mayoritariamente por mujeres. Aun así, conforme avanzan los años, el porcentaje de hombres aumenta. De esta manera, el crecimiento de matrícula ha favorecido la entrada de estudiantes varones y equilibra la composición del estudiantado. Este predominio de alumnas es una característica compartida con la regionalización de la UCR, pues, para este mismo periodo, la mayoría de los estudiantes de sus sedes regionales son mujeres. ${ }^{23}$

Ahora pasemos a caracterizar a los educandos según su grupo de edad. En 1976, la distribución de los grupos de edad indica que 14,2\% de los alumnos tenía 19 años o menos, 35,3 \% estaba entre 20 y 24 años, $27,2 \%$ entre 25 y 29 años y el restante $23,3 \%$ tenía 30 años o más. ${ }^{24}$ Estos datos revelan que, en los primeros años de funcionamiento, la institución fue más atrayente para estudiantes ya entrados en sus 20 . Además, $41,1 \%$ de los alumnos estaba casado, lo que hace suponer que son personas que ya trabajaban para mantener sus responsabilidades familiares. ${ }^{25}$

Algo similar ocurrió en las sedes de la UCR en San Ramón, Liberia y Limón, donde los primeros estudiantes fueron principalmente docentes que ya

23 Universidad de Costa Rica, Acta del Consejo Universitario 5760, 52.

24 Consejo Nacional de Rectores (CONARE), Estadística de la Educación Superior (San José, Costa Rica: CONARE, 1978), 62.

25 Ibíd., 63. 
laboraban y buscaban especializarse. ${ }^{26}$ Esto significa que eran mayores de edad y casados. Al ser la Escuela Normal de Pérez Zeledón la base de la Sede Regional Brunca y considerando que inició orientada a carreras educativas, ¿pudo presentar un comportamiento similar al mostrado en las sedes de la UCR? ¿Las edades reportadas en 1976 sugieren que se trataba de personas insertas en el mercado laboral en búsqueda de especialización? Son interrogantes para ser abordadas en futuras investigaciones.

La composición por grupos de edades para los estudiantes de las dos primeras décadas del siglo XXI se observa en el siguiente cuadro:

Cuadro 2. Sede Regional Brunca. Distribución porcentual de la matrícula por grupo de edad (2007-2018)

\begin{tabular}{|l|c|c|c|c|c|c|}
\hline & $\mathbf{2 0}$ y menos & $\mathbf{2 1 - 2 5}$ & $\mathbf{2 6 - 3 0}$ & $\mathbf{3 1 - 3 5}$ & $\mathbf{3 6 - 4 0}$ & $\begin{array}{l}\text { Más de } \\
\mathbf{4 0}\end{array}$ \\
\hline $\mathbf{2 0 0 7}$ & 31,8 & 47,9 & 11,4 & 3,7 & 1,7 & 3,4 \\
\hline $\mathbf{2 0 0 8}$ & 35,1 & 47,7 & 10,9 & 2,7 & 1,4 & 2,1 \\
\hline $\mathbf{2 0 0 9}$ & 37,3 & 45,0 & 11,4 & 2,6 & 1,9 & 1,7 \\
\hline $\mathbf{2 0 1 0}$ & 39,6 & 42,4 & 12,6 & 2,6 & 1,4 & 1,2 \\
\hline $\mathbf{2 0 1 1}$ & 38.9 & 44,1 & 11,5 & 2,5 & 1,6 & 1,4 \\
\hline $\mathbf{2 0 1 2}$ & 37,8 & 46,6 & 10,5 & 2,4 & 1,5 & 1,1 \\
\hline $\mathbf{2 0 1 3}$ & 38,3 & 47,0 & 10,0 & 2,6 & 0,9 & 1,1 \\
\hline $\mathbf{2 0 1 4}$ & 36,1 & 50,0 & 9,8 & 2,5 & 0,9 & 0,9 \\
\hline $\mathbf{2 0 1 5}$ & 38,1 & 50,0 & 8,5 & 2,1 & 0,8 & 0,6 \\
\hline $\mathbf{2 0 1 6}$ & 37,2 & 51,1 & 7,8 & 2,5 & 0,7 & 0,7 \\
\hline $\mathbf{2 0 1 7}$ & 35,3 & 52,1 & 8,4 & 2,9 & 0,6 & 0,7 \\
\hline $\mathbf{2 0 1 8}$ & 37,2 & 50,3 & 8,3 & 2,4 & 1,1 & 0,6 \\
\hline
\end{tabular}

Fuente: Elaborado a partir de datos de la Oficina de Registro UNA. Matrícula Sede Brunca 2007-2018.

La información anterior indica que la expansión de matrícula experimentada en este periodo se traduce en una comunidad de estudiantes cada vez más joven. Este fenómeno es más pronunciado en el grupo de 20 años y menos; coincide con el aumento de alumnos nuevos admitidos cada año. Además, como se detalla más adelante, el avance de los años en este periodo también tiene una incidencia en la titulación: disminuyen las licenciaturas y aumentan tanto los

26 Edgar Chavarría Solano, «La docencia en la Sede de Guanacaste», Manuscrito inédito (Costa Rica: Departamento de Docencia Universitaria, Universidad de Costa Rica, 2001), 11. 
bachilleratos como los diplomados. Este comportamiento pudo afectar la composición por edades, al disminuir los estudiantes que optaban por permanecer más años para obtener una licenciatura y, en cambio, aumentar la cantidad de aquellos que en menos años obtienen un título y concluyen, al menos en esta sede, sus estudios.

Otro aspecto socioeconómico fundamental en este estudio es el relacionado con la procedencia geográfica de los alumnos, tras identificar, primeramente, su origen cantonal. En 1976, de 309 alumnos matriculados en la sede, 96,8 \% residían en la provincia de San José y el resto provenía de Puntarenas. ${ }^{27}$ Es de suponer que estos residentes josefinos serían originarios del cantón de Pérez Zeledón y una representación significativamente menor de estudiantes venía de los cantones puntarenenses de la Región Brunca. Aunque no contamos con datos estadísticos de las décadas siguientes, esta pudo ser una tendencia que se mantuvo a lo largo de los años, pues un integrante del gobierno juzgaba en 1984 que «La queja que existe contra el Centro Regional de San Isidro de Pérez Zeledón se basa principalmente en el hecho que ha venido a resolver el problema parcialmente, pues los estudiantes que asisten a esta unidad académica proceden en un porcentaje muy elevado del centro de Pérez Zeledón». ${ }^{28}$

Este panorama nos hace preguntar: ¿una matrícula menor se concentró en estudiantes procedentes de Pérez Zeledón? ¿Qué sucedió en los periodos de expansión? No contamos con datos que permitan analizar lo ocurrido en las décadas de 1980 y 1990 . No obstante, la información disponible posibilita estudiar el último periodo de expansión registrado, a partir del 2007. La distribución del estudiantado, tomando en consideración sus cantones de origen, se muestra en el siguiente gráfico:

Gráfico 5. Sede Regional Brunca. Distribución porcentual de la matrícula por cantón de procedencia (2007-2018)

27 CONARE, 1978, 64.

28 UCR, Acta del Consejo Universitario 3129, 6. El subrayado es mío. 


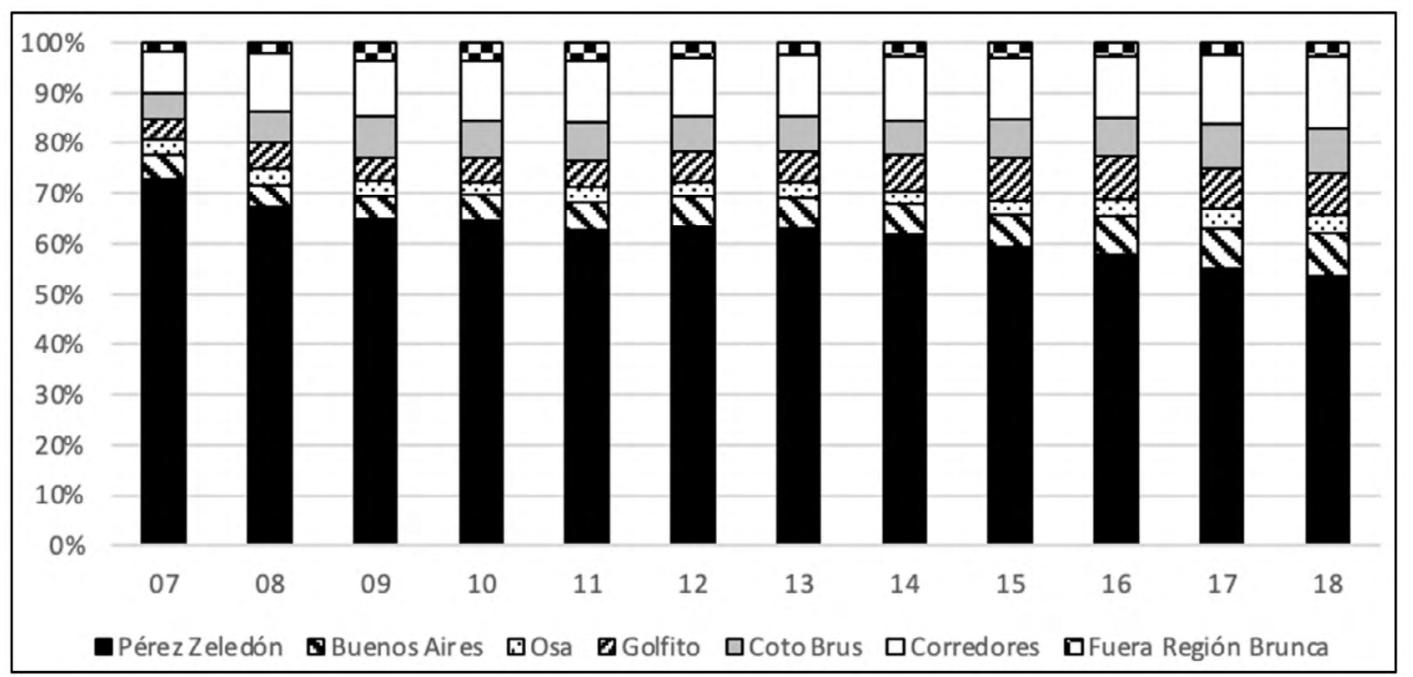

Fuente: Elaborado a partir de datos de la Oficina de Registro UNA, Matrícula Sede Brunca 2007-2018.

El primer dato que resalta es que la gran mayoría de estudiantes proviene de la Región Brunca. Esto muestra el impacto inmediato que realiza la institución en su zona de influencia, pues beneficia, en gran manera, alumnos de su región. Añadido a lo anterior, el cantón más representado es Pérez Zeledón, aunque, conforme avanza el periodo estudiado, su porcentaje va disminuyendo, sin abandonar nunca su posición privilegiada. De esta manera, al aumentar la matrícula, los educandos que ingresan pertenecen cada vez más a los otros cantones de la Región Brunca. La existencia del Campus Coto alimenta en parte el fenómeno, dado que favorece el incremento de representación de los cantones fronterizos Corredores, Coto Brus y Golfito. Así, en el 2007, el 78,4 \% de los estudiantes de estos tres cantones concentraba su matrícula en el Campus Coto, cifra que se acrecienta a 92,7\% en el 2018. En especial, Corredores amplía, significativamente, su participación y en todo el periodo es el segundo cantón más representado.

Para observar la distribución distrital de estos mismos alumnos, se decidió mostrar a los procedentes de la cabecera de su cantón y a los originarios de un distrito distinto a la cabecera cantonal. Este análisis arroja los siguientes resultados:

Gráfico 6. Sede Regional Brunca. Distribución porcentual de la matrícula por distrito de procedencia (2007-2018) 


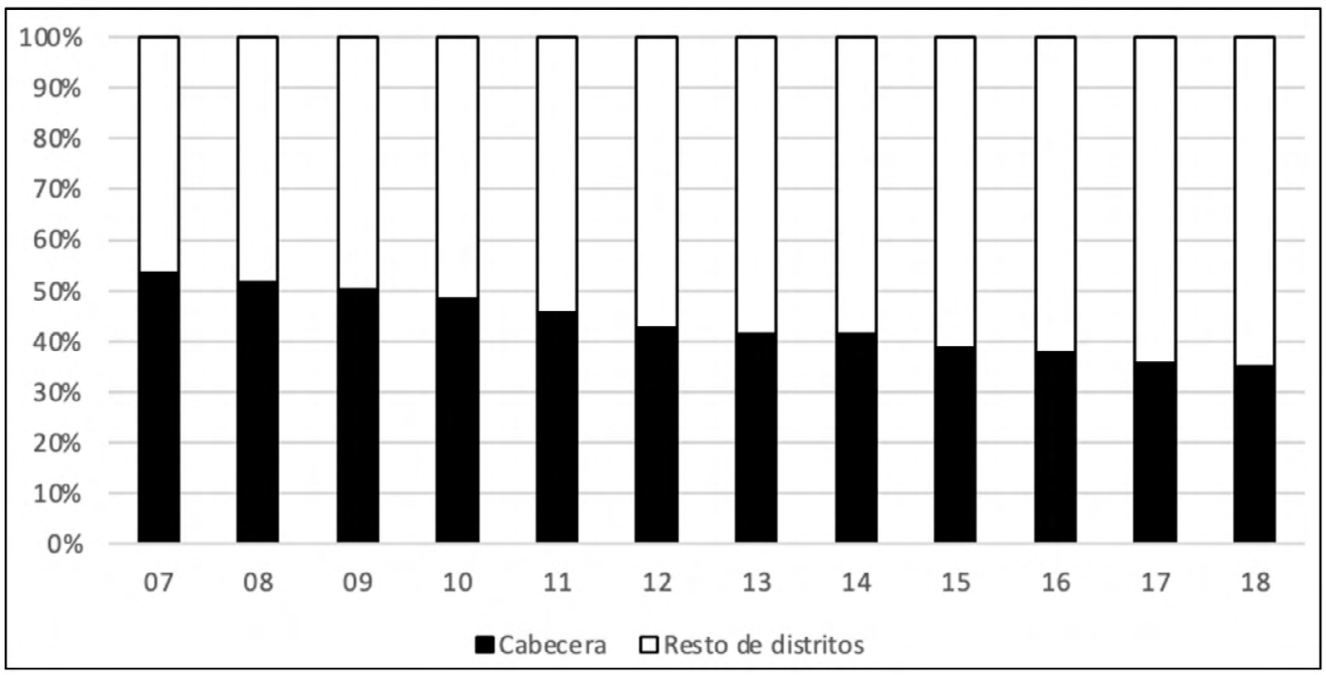

Fuente: Elaborado a partir de datos de la Oficina de Registro UNA, Matrícula Sede Brunca 2007-2018.

El aumento de la matrícula y la mayor representación de estudiantes por cantón también ha tenido un efecto en su distribución distrital, con un crecimiento significativo de aquellos alumnos provenientes de un distrito distinto a la cabecera cantonal. Además, los dos cantones más representados en el estudiantado son, a su vez, los dos que presentaron cambios más notables en su composición distrital. De esta manera, Corredores pasa de 27,4\% a 57,2 \% de alumnos pertenecientes a distritos distintos al central. Tal incremento quiere decir que el Campus Coto, principal receptor de alumnos corredoreños, atrajo, primordialmente, a educandos de distritos distintos a Corredores, su cabecera. En el caso de Pérez Zeledón, este pasa de 45,3 \% a 65,7 \% de alumnos residentes fuera de la cabecera cantonal. Este cambio afecta, principalmente, a la población del Campus Pérez Zeledón, donde matriculan la mayoría de los estudiantes del cantón josefino.

En cuanto a la distribución por zona, tenemos los siguientes resultados: 
Gráfico 7. Sede Regional Brunca. Distribución total de la matrícula por zona de procedencia (2007-2018)

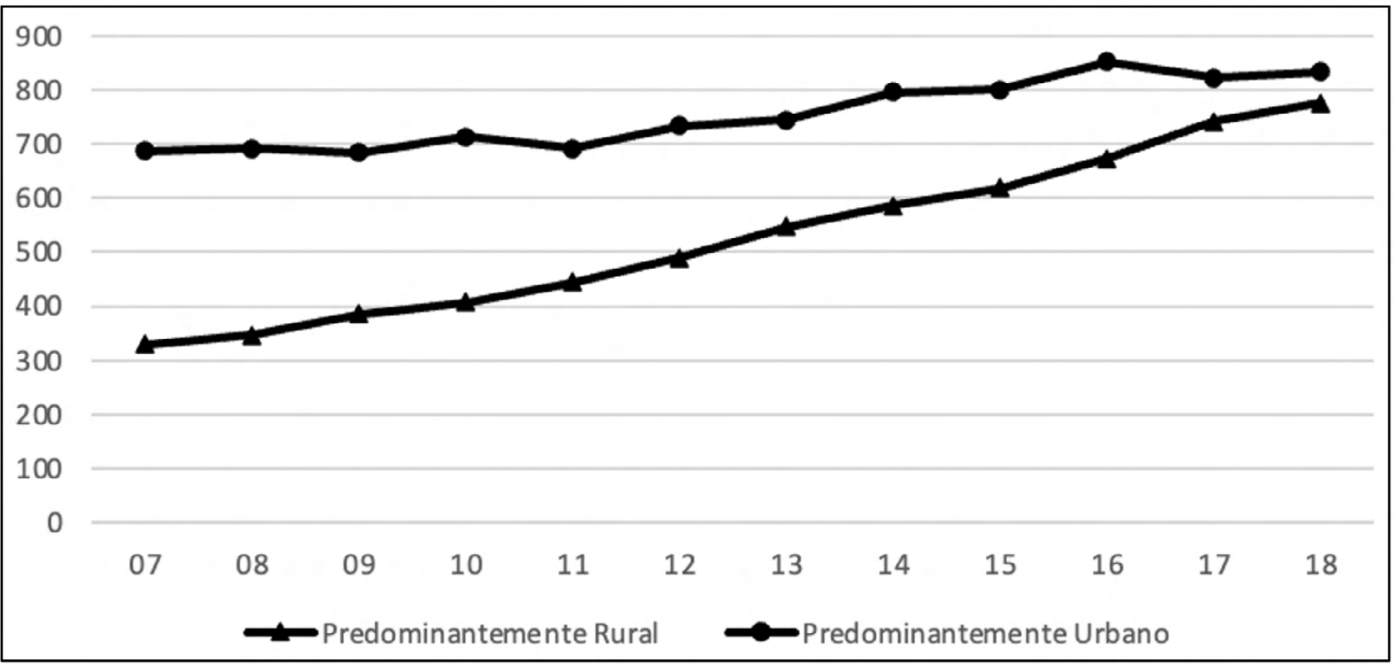

Fuente: Elaborado a partir de datos de la Oficina de Registro UNA, Matrícula Sede Brunca 2007-2018.

La diversificación antes descrita de la procedencia geográfica de los estudiantes tiene otra consecuencia importante y es la mayor participación de alumnos provenientes de zona rural. Para el 2007, estos representaban el 32,4 $\%$ del total de matriculados, cifra que asciende hasta el 48,2 \% en el 2018. Todo apunta a que el agrandamiento de la participación de cantones distintos a Pérez Zeledón y el aumento de estudiantes provenientes de distritos distintos a la cabecera cantonal significó una mayor representación de zona rural. Así, la expansión de matrícula registrada desde el 2007 implica el ingreso masivo de estudiantes rurales, hasta llegar a ser casi la mitad de la comunidad estudiantil una década después.

La relación establecida entre la zona geográfica originaria del estudiante y su condición socioeconómica se determina por medio del IDS, que calcula el nivel de desarrollo de cada distrito. En la población de la Sede Regional Brunca, aplicando ese índice, obtenemos el siguiente resultado: 
Gráfico 8. Sede Regional Brunca. Distribución porcentual de la matrícula por Índica de Desarrollo Distrital (IDS) ${ }^{\mathrm{a}}(2007-2018)$

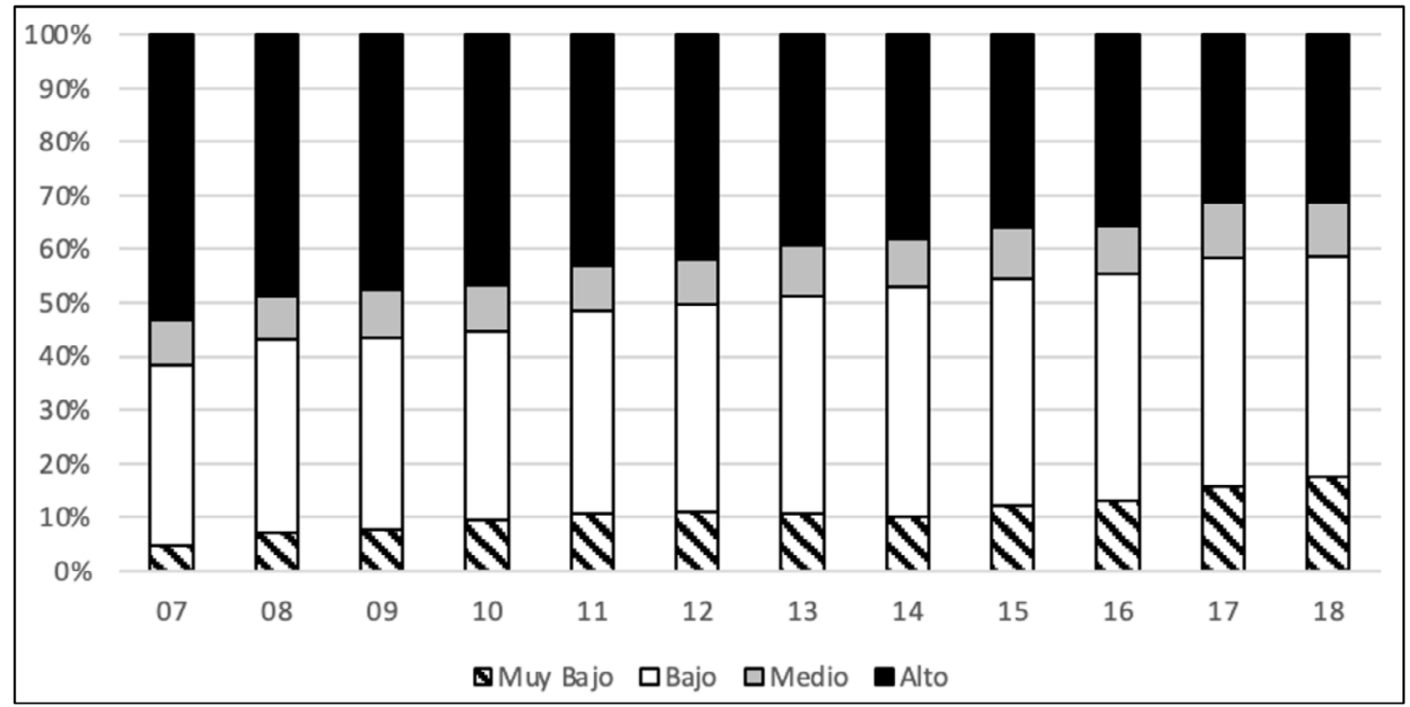

Nota: ${ }^{\text {a }}$ Se tomó como base el IDS de 2017.

Fuente: Elaborado a partir de datos de Oficina de Registro UNA, Matrícula Sede Brunca 2007-2018.

Los datos indican que otra consecuencia de esta diversificación geográfica es la mayor participación de estudiantes provenientes de distritos con un IDS muy bajo o bajo. Así, observamos que al inicio del periodo en estudio los alumnos que habitan distritos con un IDS alto encarnan más de la mitad. En este año, recordemos, la representación de Pérez Zeledón, cabeceras cantonales y zona urbana es mayor. Contrariamente, al aumentar la participación de los otros cantones de la Región Brunca, de distritos distintos a la cabecera cantonal y de la zona rural aumenta considerablemente el porcentaje de IDS bajo y muy bajo. Este aumento, se debe señalar, se realiza en un contexto de crecimiento de la matrícula, con lo cual dicha expansión significa la entrada de estudiantes que se desenvuelven en contextos geográficos menos desarrollados, más desiguales y con mayores índices de pobreza.

Otro indicador importante es el referido a la condición económica de los alumnos. En lo relacionado con el ingreso de los hogares, en 1976 el 41,7 \% de los estudiantes de la Sede Regional Brunca provenían de hogares con una entrada monetaria de 2000 colones o menos ${ }^{29}$, lo que equivale a 124,7 dólares de $1970^{30}$. Este dato es mayor que la media del total de estudiantes de la UNA para dicho

29 CONARE, 1978, 65.

30 Este cálculo lo tomo de Molina Jiménez, La educación..., 405. 
año, ubicado en $38,8 \%{ }^{31}$ Además, indica que la mayoría de los alumnos de esta sede venía de hogares con un ingreso superior a la distribución del ingreso promedio del país. ${ }^{32}$ Recordando lo referido en el estudio de los grupos de edad, el aspecto informativo económico puede reforzar la sospecha de que estos primeros alumnos de la sede ya laboraban y generaban sus propios ingresos.

No poseemos datos financieros para el resto de las décadas, pero contamos con un indicador que puede darnos pistas acerca de las condiciones económicas de los hogares de los estudiantes. Este es el porcentaje de quienes son becados, pues uno de los criterios para entregar una beca es justamente la cantidad de ingreso del grupo familiar del alumno. Los datos disponibles son escasos para las décadas de 1980 y 1990, así como más completos para el siglo XXI. Estos se muestran en el siguiente gráfico:

Gráfico 9. Sede Regional Brunca. Distribución porcentual de la matrícula por condición de beca (1981-2018)

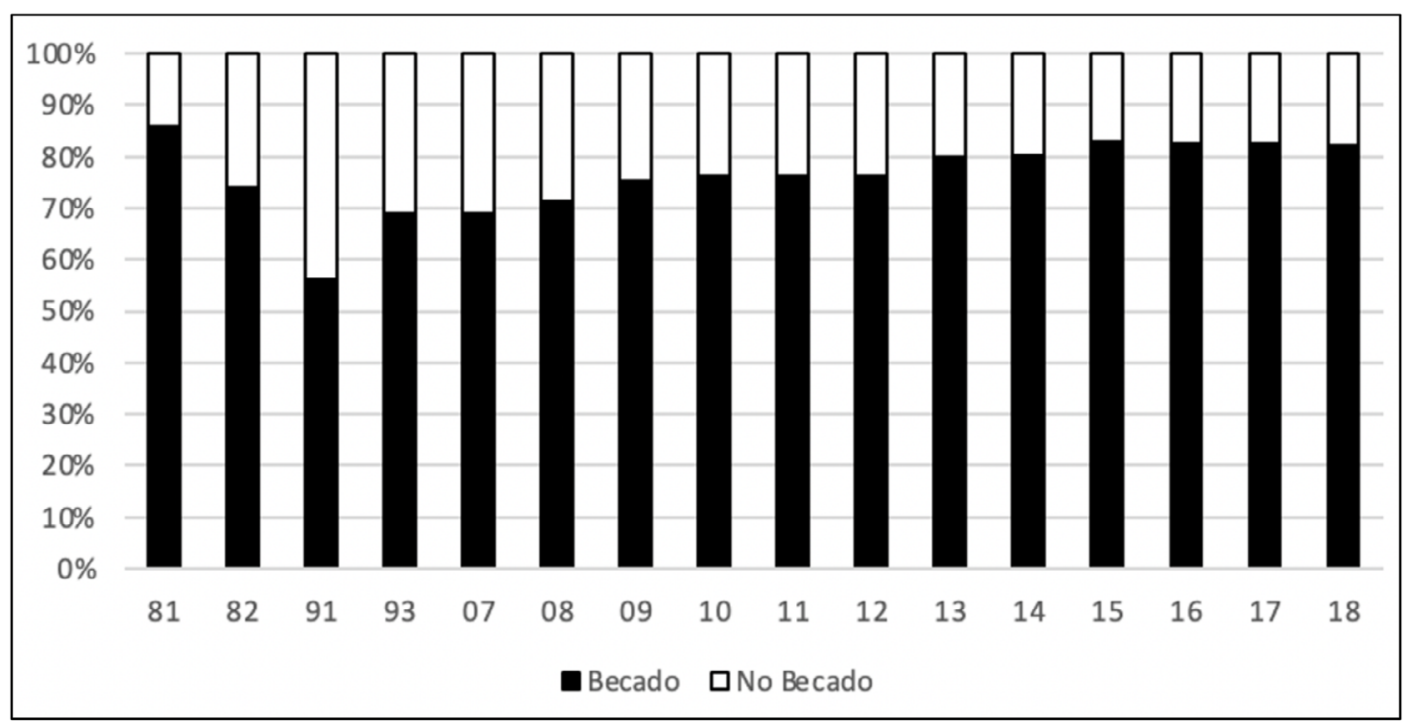

Fuente: Elaborado a partir de datos de CONARE, Estadística de la Educación Superior, 1982, cuadro 1.5 .3 , pág. $67 ; 1983$, cuadro 1.6.4, pág. 90; 1992, cuadro 1.5.8, pág. 132; 1996, cuadro 1.5.7, pág. 187. Oficina de Registro UNA, Matrícula Sede Brunca 2007-2018.

31 CONARE, 1978, 65 .

32 Nuevamente utilizo los cálculos de Molina Jiménez, La educación..., 406. 
En primer lugar, es interesante destacar el alto porcentaje de estudiantes becados en 1981 y 1982, lo que coincide con los dos años en los cuales la crisis económica fue más aguda. Esto se tradujo en una baja considerable en los ingresos de los hogares costarricenses, hecho que es probable afectara los hogares de los estudiantes de la sede en esa época. Posteriormente, se registra una baja en 1991 y un incremento en 1993, aunque con un porcentaje todavía menor al registrado en esos dos años iniciales de la década de 1980.

Con respecto al 2007 y en adelante, al ingresar una mayor cantidad de estudiantes provenientes de zona rural y de distritos con IDS muy bajo o bajo, también aumenta la cantidad de aquellos que requieren una beca para continuar con sus estudios. Así, el porcentaje becado sube un poco más de 10 puntos porcentuales entre el 2007 y el 2018. Con esto se deduce que el aumento en matrícula para este periodo se traduce en la entrada de una mayor cantidad de educando que necesitan los servicios de exoneración, asistencia económica y demás beneficios otorgados por el sistema de becas, para poder concluir su plan de estudios.

\section{Conformación de la comunidad de graduados}

En la tercera sección del artículo, se particulariza a la comunidad de graduados que ha producido esta sede. Esto brinda pistas sobre las oportunidades académicas ofrecidas por esta universidad en la región y el grado de especialización que pueden alcanzar los estudiantes que acuden a esta sede.

El primer aspecto en ser estudiado es el total de títulos otorgados por año y su distribución por grado. Esta información se expone en el siguiente gráfico: 
Gráfico 10. Sede Regional Brunca. Distribución total de títulos por grado (1980-2018)

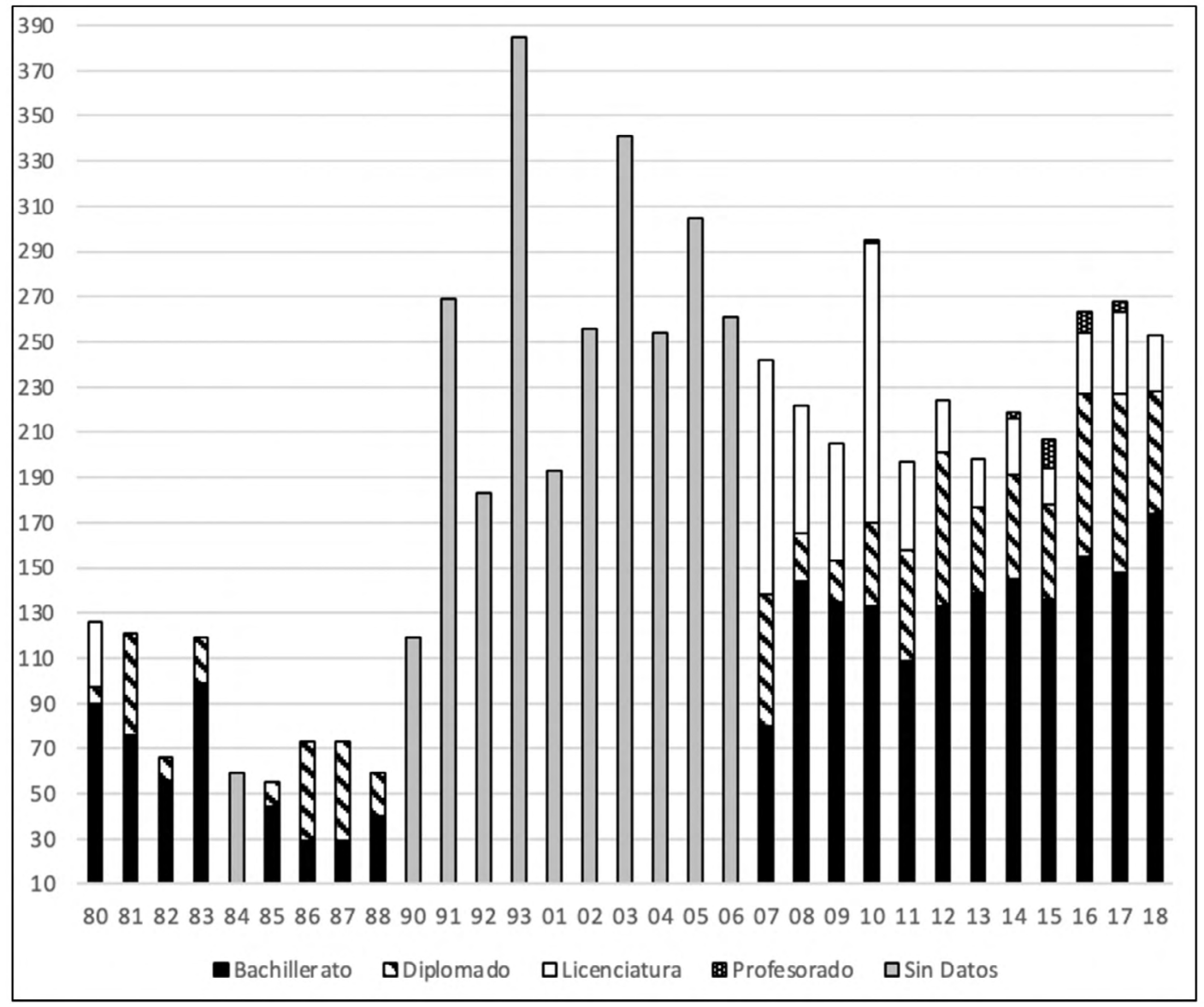

Fuente: Elaborado a partir de datos de CONARE, Estadística de la Educación Superior, 1983 cuadro 1.4 .6 , pág. $70 ; 1984$, cuadro 1.3 .5 , pág. 77; 1985, cuadro 1.3 .5 , pág. $60 ; 1987$, cuadro 1.3.9, pág. $73 ; 1988$, cuadro 1.3.9, pág. 79; 1989, cuadro 1.3.11, pág. 84. Cheng, et al.,1994, cuadro 16, pág. 217. Oficina de Registro UNA, cuadro 3. Estudiantes graduados por año, según sede y facultad 2001-2015. Oficina de Registro UNA, Graduación Sede Brunca 2007-2018.

Lo primero por observar es la cantidad de títulos generados en todo el periodo, la cual varía notablemente durante todos los años registrados. La suma 
menor de títulos se entregó en la década de 1980, pues se contaba con una población estudiantil más pequeña.

Con respecto a los grados, el bachillerato está presente en todos los años en los cuales hay datos. El segundo grado presente en todo el periodo es el diplomado, que a partir del 2010 aumenta su cantidad. Así, estos dos tipos de títulos son los más otorgados por la institución. La licenciatura, en la década de 1980, solamente se encuentra en el primer año; a partir de 2007, está presente en todos los años, aunque desde el 2012 su cantidad disminuye. Finalmente, se debe mencionar que esta institución nunca ha dado títulos de posgrado.

La distribución de los graduados por sexos arroja los siguientes resultados:

Gráfico 11. Sede Regional Brunca. Distribución porcentual de títulos por sexo (2007-2018)

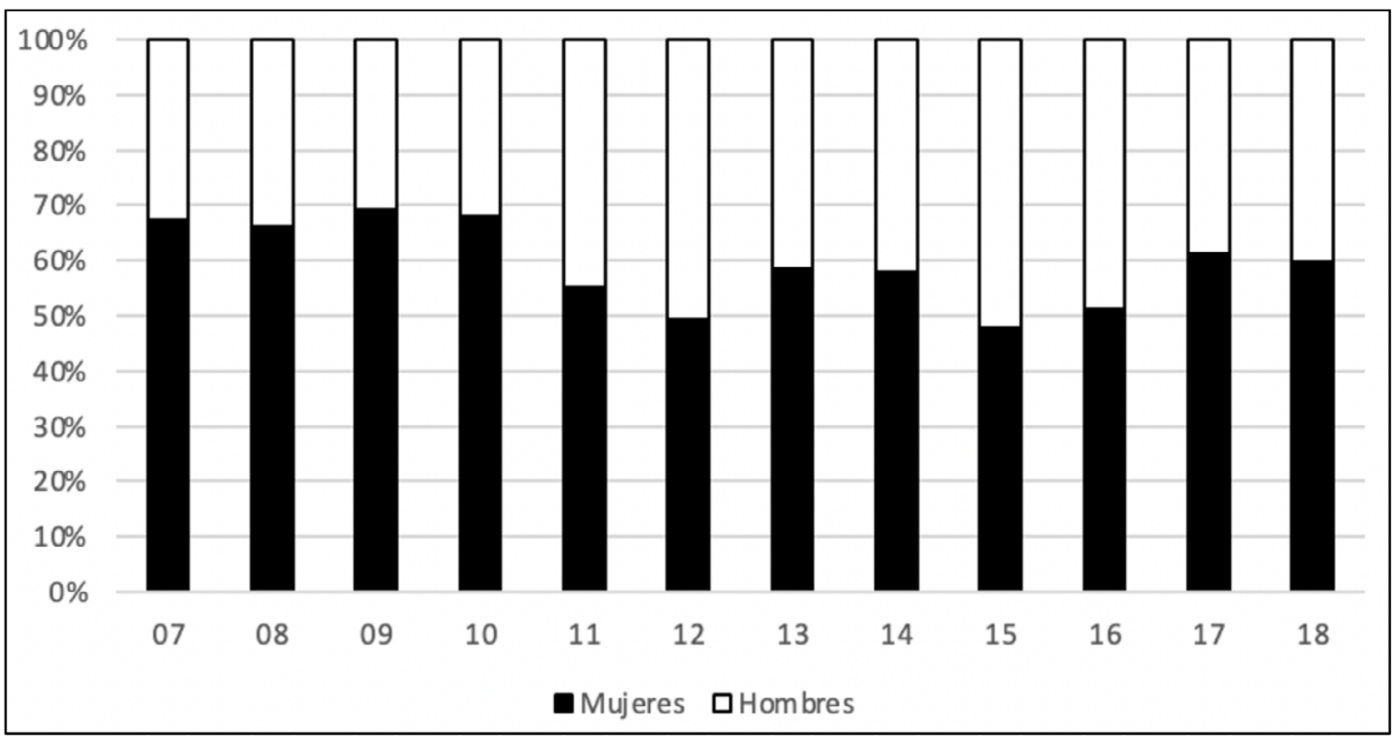

Fuente: Oficina de Registro UNA. Graduación Sede Brunca 2007-2018.

En general, en el periodo analizado, la mayoría de los títulos se ha entregado a mujeres, aunque esta tendencia ha bajado conforme avanzan los años, cediendo ante la titulación de hombres. Aun así, las féminas nunca abandonan su porcentaje mayoritario.

Finalmente, en la distribución de los títulos por área temática, tenemos los siguientes resultados:

Cuadro 3. Sede Regional Brunca. Distribución porcentual de títulos por área 
temática (1985-2018)

\begin{tabular}{|l|c|c|c|c|c|}
\hline & Letras & Turismo & $\begin{array}{l}\text { Ciencias e } \\
\text { Ingeniería }\end{array}$ & Educación & $\begin{array}{l}\text { Sociales y } \\
\text { Economía }\end{array}$ \\
\hline $\mathbf{1 9 8 5}$ & & & & 80,0 & \\
\hline $\mathbf{1 9 8 6}$ & & & & 70,0 & \\
\hline $\mathbf{1 9 8 7}$ & & & & 70,0 & \\
\hline $\mathbf{1 9 8 8}$ & & & & 100,0 & \\
\hline $\mathbf{2 0 0 7}$ & & & 16,5 & 64,5 & 19,0 \\
\hline $\mathbf{2 0 0 8}$ & & & 17,1 & 70,3 & 12,6 \\
\hline $\mathbf{2 0 0 9}$ & 0,4 & & 13,7 & 69,8 & 16,1 \\
\hline $\mathbf{2 0 1 0}$ & 5,7 & 11,8 & 13,9 & 52,7 & 15,9 \\
\hline $\mathbf{2 0 1 1}$ & 5,6 & 15,2 & 28,9 & 31,5 & 18,8 \\
\hline $\mathbf{2 0 1 2}$ & 9,8 & 18,8 & 35,3 & 16,1 & 20,0 \\
\hline $\mathbf{2 0 1 3}$ & 8,7 & 25,2 & 23,7 & 10,1 & 32,3 \\
\hline $\mathbf{2 0 1 4}$ & 6,8 & 11,4 & 30,2 & 8,7 & 42,9 \\
\hline $\mathbf{2 0 1 5}$ & 7,7 & 8,2 & 31,4 & 25,6 & 27,1 \\
\hline $\mathbf{2 0 1 6}$ & 9,5 & 13,4 & 31,9 & 14,8 & 30,4 \\
\hline $\mathbf{2 0 1 7}$ & 13,8 & 17,2 & 29,8 & 13,1 & 26,1 \\
\hline $\mathbf{2 0 1 8}$ & 6,7 & 13,4 & 20,1 & 13,0 & 37,5 \\
\hline
\end{tabular}

Fuente: Elaborado a partir de datos de CONARE, Estadística de la Educación Superior, 1987, cuadro 1.3 .9 , pág. $73 ; 1988$, cuadro 1.3 .9 , pág. $79 ; 1989$, cuadro 1.3 .11 , pág. 84. Oficina de Registro UNA, Graduación Sede Brunca 2007-2018.

La importancia de los títulos en carreras de educación es muy notoria en la década de 1980. Sumada a los datos del cuadro 1, se refuerza la sospecha del uso de la regionalización universitaria como herramienta para titular docentes y así enfrentar la crisis histórica en la formación de este tipo de profesionales en el país. Considerando que los grados entregados en estos años fueron diplomados y bachilleratos - ver gráfico 10-, se vislumbra una posible estrategia de calificar rápidamente a especialistas en educación. Esta misma táctica fue utilizada en sedes regionales de la UCR en Turrialba, Liberia y Limón, donde se firmaron convenios con el Ministerio de Educación Pública (MEP) para titular, en corto tiempo, a maestros y así enfrentar su creciente demanda. ${ }^{33}$

33 Albert Espinoza Sánchez y Rosa Rosales Ortiz, «Tendencia de graduación en la Sede Guanacaste de la Universidad de Costa Rica», InterSedes, vol. 2, n. ${ }^{\circ}$ 2-3 (2001): 126 https://revistas.ucr.ac.cr/index.php/ intersedes/article/view/774/835; Ivone Lepe Jorquera, «La Sede del Caribe: experiencia de su desarrollo en el periodo 2002-2010» (Ponencia presentada en el I Congreso de Regionalización de la Universidad de Costa Rica, Costa Rica, 17, 18 y 19 de abril de 2018), 8. 
En el siglo XXI, entre el 2007 y el 2010, las carreras de educación tienen un peso muy significativo en los títulos otorgados. Esto empieza a disminuir en el 2011, ligado a la diversificación temática en la oferta académica registrada en la sede. Además, observando nuevamente los datos del gráfico 10, existe una relación entre la diversificación temática, la disminución de licenciaturas y el aumento de bachilleratos y diplomados. Los títulos en educación fueron los que dominaron el grado de licenciatura entre el 2007 y el 2010; al disminuir su peso en la titulación, también se aminoró su peso en el grado concedido. Las nuevas áreas temáticas entregaron, principalmente, títulos en diplomado y bachillerato; destacan diplomados en Inglés y Programación, así como bachilleratos en Turismo, Administración y Sistemas de Información.

\section{Conclusiones}

El programa de regionalización de la UNA está en sintonía con el carácter democratizante que configura a esta universidad estatal. Así, desde su Estatuto Orgánico, dicha casa de estudios busca expandir el acceso a educación superior en la sociedad costarricense. Un estudio de la comunidad estudiantil beneficiada con este proceso en la Sede Regional Brunca aporta algunas pistas para medir como ha sido implementada esa visión democrática.

La descripción de la trayectoria de la matrícula general de la UNA sugiere que esta se encuentra condicionada por el ambiente político y económico del país. Lo anterior se refleja en su programa de regionalización, pues periodos con un clima favorable se traducen en una expansión de matrícula en sedes regionales. Contrariamente, lapsos de estrechez tienen como consecuencia una reducción de la cantidad de estudiantes regionalizados.

Concretamente en el caso de la Sede Regional Brunca, el trayecto de su matrícula evidencia que el proceso de consolidación de este centro de estudios fue complejo. En sintonía con lo descrito, el crecimiento más sostenido ocurre a partir de 2007; coincide con un ambiente político y económico favorable para las universidades públicas.

La caracterización del gremio estudiantil beneficiado con que opere la Sede Regional Brunca muestra que el expandir la matrícula significa una diversificación importante en la composición de los alumnos. Los datos fragmentarios de las décadas 1970 y 1980 permite al menos sospechar que una matrícula reducida se concentra en personas provenientes del cantón de Pérez Zeledón. Con la ampliación registrada a partir de 2007, se produce un mayor acceso de estudiantes originarios de los restantes cantones de la Región Brunca. A su vez, este crecimiento se traduce en una admisión mayor de alumnos pertenecientes a grupos sociales vulnerables. De esta manera, la segunda década del siglo XXI ha significado más participación de mujeres, la zona rural, los cantones fronterizos y los hogares ubicados en distritos con desarrollo social bajo. 
La particularización realizada en la comunidad de graduados de la sede muestra que el énfasis de esta institución ha sido colocado en las carreras cortas. Además, la diversificación temática de la oferta académica ha sido un proceso pausado. La información fragmentada que se posee para 1980 sugiere que el enfoque estaba en carreras de educación, lo que limitaba las aspiraciones vocacionales de los estudiantes que accedían a esta sede. Para el 2007, esta tendencia se mantenía, aunque a partir de ese año inicia un proceso de diversificación. Este ha abarcado más áreas temáticas, pero concentrado en pocas carreras por área. Tal diversificación ha sido realizada en sintonía con el crecimiento en matrícula experimentado por la sede en esta época.

La conclusión de este trabajo es que la misión democratizante de la UNA, manifestada en la Sede Regional Brunca, está condicionada por el ambiente político y económico del país. En periodos favorables, como el registrado a partir de 2007 , se ha observado un fortalecimiento de esta institución y un robustecimiento de su misión democratizadora. Esto se demuestra en la expansión de su matrícula, la cual se ha traducido en un acceso mayor de grupos sociales vulnerables a los servicios educativos brindados por la universidad. Igualmente, este fortalecimiento institucional ha significado una diversificación en la oferta académica, lo que brinda mayores opciones vocacionales a las personas de la región explorada.

\section{Referencias}

Centro Universitario de San Ramón, Informe de Labores 1975-1976. Manuscrito inédito, s. f.

Consejo Nacional de Rectores (CONARE). Matrícula del primer periodo lectivo de las instituciones de educación superior universitaria estatal, por institución y sede. 2000-2017. https://www.conare.ac.cr/servicios/estadistica?download=144:cifras-relevantes-de-la-educacion-superior-2000-2016-junio-2016.

Consejo Nacional de Rectores (CONARE). Estadística de la Educación Superior. San José, Costa Rica: CONARE, 1978.

Consejo Nacional de Rectores (CONARE). Estadística de la Educación Superior 1978. San José, Costa Rica: CONARE, 1980.

Consejo Nacional de Rectores (CONARE). Estadística de la Educación Superior 1979. San José, Costa Rica: CONARE, 1980.

Consejo Nacional de Rectores (CONARE). Estadística de la Educación Superior 1980. San José, Costa Rica: CONARE, 1981.

Consejo Nacional de Rectores (CONARE). Estadística de la Educación Superior 1981. San José, Costa Rica: CONARE, 1982. 
Consejo Nacional de Rectores (CONARE). Estadística de la Educación Superior 1982. San José, Costa Rica: CONARE, 1983.

Consejo Nacional de Rectores (CONARE). Estadística de la Educación Superior 1983. San José, Costa Rica: CONARE, 1984.

Consejo Nacional de Rectores (CONARE). Estadística de la Educación Superior 1984. San José, Costa Rica: CONARE,1985.

Consejo Nacional de Rectores (CONARE). Estadística de la Educación Superior 1985. San José, Costa Rica: CONARE, 1986.

Consejo Nacional de Rectores (CONARE). Estadística de la Educación Superior 1986. San José, Costa Rica: CONARE, 1987.

Consejo Nacional de Rectores (CONARE). Estadística de la Educación Superior 1987. San José, Costa Rica: CONARE, 1988.

Consejo Nacional de Rectores (CONARE). Estadística de la Educación Superior 1988. San José, Costa Rica: CONARE, 1989.

Consejo Nacional de Rectores (CONARE). Estadística de la Educación Superior 1989. San José, Costa Rica: CONARE, 1991.

Consejo Nacional de Rectores (CONARE). Estadística de la Educación Superior 1990. San José, Costa Rica: CONARE, 1991.

Consejo Nacional de Rectores (CONARE). Estadística de la Educación Superior 1991. San José, Costa Rica: CONARE, 1992.

Consejo Nacional de Rectores (CONARE). Estadística de la Educación Superior 1992. San José, Costa Rica: CONARE, 1994.

Consejo Nacional de Rectores (CONARE). Estadística de la Educación Superior 1993 y 1994. San José, Costa Rica: CONARE, 1996.

Consejo Nacional de Rectores (CONARE). Estadística de la Educación Superior 1995, 1996 y 1997. San José, Costa Rica: CONARE, 1999.

Chavarría Solano, Edgar. «La docencia en la Sede de Guanacaste». Manuscrito inédito. Costa Rica: Departamento de Docencia Universitaria, Universidad de Costa Rica, 2001.

Dachner Trujillo, Yolanda. «La Regionalización de la Universidad de Costa Rica, una propuesta para avanzar». Ponencia presentada en el IV Seminario Carlos Monge Alfaro-La universidad pública en el mundo contemporáneo, Costa Rica, 4, 5 y 6 de noviembre de 1998.

Espinoza Sánchez, Albert y Rosales Ortiz, Rosa. «Tendencia de graduación en la Sede Guanacaste de la Universidad de Costa Rica». InterSedes, vol. 2, n. ${ }^{\circ}$ 2-3 (2001): 123-134. https://revistas.ucr.ac.cr/index.php/intersedes/ article/view/774/835.

Fonseca Zúñiga, Edgardo. «Las Humanidades en la génesis de la regionalización universitaria: los Estudios Generales y el origen del proceso de regionalización de la Universidad de Costa Rica». Revista Estudios, número 
especial (2020): 1-35. https://revistas.ucr.ac.cr/index.php/estudios/article/ view/40925/41537.

Fonseca Zúñiga, Edgardo. «La regionalización de la educación superior en Costa Rica, el caso de la Universidad de Costa Rica en Golfito, 1984-2017»». Revista Estudios, n. 38 (junio-noviembre 2019): 111-134. https://revistas. ucr.ac.cr/index.php/estudios/article/view/37463/38268

Molina Jiménez, Iván. La educación en Costa Rica. Desde la época colonial hasta el presente. San José, Costa Rica: EDUPUC, 2016.

Lepe Jorquera, Ivone. «La Sede del Caribe: experiencia de su desarrollo en el periodo 2002-2010». Ponencia presentada en el I Congreso de Regionalización de la Universidad de Costa Rica, Costa Rica, 17, 18 y 19 de abril de 2018.

Marvin Cheng Ta, et al. «La política de regionalización en la educación superior estatal». Tesis de Licenciatura en Administración Pública, Universidad de Costa Rica, 1994.

Ministerio de Planificación Nacional y Política Económica (MIDEPLAN). Índice de Desarrollo Social 2017. San José, Costa Rica: MIDEPLAN, 2018.

Oficina de Registro UNA. Cuadro 3 Estudiantes graduados por año, según sede $y$ facultad 2001-2015. Manuscrito inédito, s. f.

Oficina de Registro UNA. Admisión Sede Brunca 2007-2018. Manuscrito inédito, 2019.

Oficina de Registro UNA. Graduación Sede Brunca 2007-2018. Manuscrito inédito, 2019.

Oficina de Registro UNA, Matrícula Sede Brunca 2007-2018. Manuscrito inédito, 2019.

Programa Estado de la Nación. IV Estado de la Educación. San José, PEN, 2013.

Programa Estado de la Nación. V Estado de la Educación. San José, PEN, 2015.

Universidad de Costa Rica. Acta Consejo Universitario 3129.

Universidad de Costa Rica. Acta Consejo Universitario 5760.

Universidad Nacional. Estatuto Orgánico. http://www.documentos.una. ac.cr/bitstream/handle/unadocs/6693/ESTATUTO-ORG\%c3\%81NICO-UNA-digital.pdf? sequence $=1$ \&isAllowed $=\mathrm{y}$. 